\title{
Fadiga em idosos em tratamento quimioterápico
}

\author{
Fatigue in the elderly undergoing chemotherapy \\ Fatiga en ancianos sometidos a la quimioterapia
}

\begin{abstract}
Thalyta Cristina Mansano-Schlosser', Maria Filomena Ceolim'
' Universidade Estadual de Campinas, Faculdade de Ciências Médicas, Programa de Pós Graduação em Enfermagem. Campinas-SP, Brasil.
\end{abstract}

Submissão: 08-07-2012 Aprovação: 16-06-2014

\section{RESUMO}

Pesquisa com delineamento transversal, realizada de agosto a dezembro de 2010, que objetivou identificar a presença e intensidade da fadiga em 140 idosos (média de idade 69,8 anos, 52,1\% do sexo feminino) com diagnóstico de câncer, em tratamento quimioterápico ambulatorial. Utilizou-se um questionário para caracterização sociodemográfica e clínica e a Escala de Fadiga de Piper-revisada. Identificou-se a presença de fadiga em 60 idosos $(42,9 \%)$ e a causa atribuída foi o câncer $(64,1 \%)$, a quimioterapia $(32,8 \%)$ e o tratamento $(3,1 \%)$. Como fator de alívio, os idosos entrevistados relataram "dormir", "rezar" e "morrer", enquanto $45 \%$ deles disseram "não ter o que fazer", pois a fadiga não poderia ser aliviada. Espera-se que este estudo alerte os profissionais de enfermagem para a relevância da fadiga e os estimule a identificar e realizar intervenções de enfermagem que visem o adequado manejo desse sintoma no paciente idoso oncológico.

Descritores: Fadiga; Câncer; Idoso; Enfermagem.

\section{ABSTRACT}

This is a cross-sectional survey conducted from August to December 2010, which aimed to identify the presence and severity of fatigue in 140 elderly (mean age 69.8 years, $52.1 \%$ female) diagnosed with cancer undergoing chemotherapy in an outpatient unit. It was used a questionnaire for sociodemographic and clinical data and Piper Fatigue Scale-revised. The presence of fatigue was identified in 60 elderly $(42.9 \%)$ and the attributed cause was cancer $(64.1 \%)$, chemotherapy $(32.8 \%)$ and treatment $(3.1 \%)$. As relief factors, the elderly respondents reported "sleeping", "pray" and "dying", while $45 \%$ of them said "there was nothing to do" because fatigue could not be relieved. We hope that this study will alert the nurses to the relevance of fatigue and encourage them to identify and carry out nursing interventions aimed at proper management of this symptom in elderly cancer patient.

Key words: Fatigue; Cancer; Elderly; Nursing.

\section{RESUMEN}

Estudio transversal, realizado entre agosto y diciembre de 2010, que objetivó identificar la presencia y severidad de fatiga en 140 ancianos (edad media 69,8 años, 52,1\% mujeres) con diagnóstico de cáncer en quimioterapia ambulatoria. Se utilizó un cuestionario para datos sociodemográficos y clínicos y la Escala de Fatiga de Piper-revisada. Fue identificada presencia de fatiga en 60 ancianos $(42,9 \%)$ y se atribuyó la causa al cáncer $(64,1 \%)$, quimioterapia $(32,8 \%)$ y tratamiento $(3,1 \%)$. Como factor de alivio, los encuestados informaron "dormir", "rezar" y "morir", mientras que $45 \%$ de ellos dijo que "no hay lo que hacer", porque la fatiga no podía ser aliviada. Se espera que este estudio contribuya a alertar a las enfermeras sobre la relevancia de la fatiga y animarles a identificar y llevar a cabo intervenciones de enfermería dirigidos a la gestión adecuada de este síntoma en los pacientes de cáncer de edad avanzada.

Palabras clave: Fatiga; Cáncer; Ancianos; Enfermería. 


\section{INTRODUÇÃO}

O avanço da tecnologia na área de saúde tem possibilitado o aumento da longevidade, de maneira que, em 2009, os resultados da Pesquisa Nacional de Amostra por Domicílio (PNAD) apontavam para um total de 11,3 milhões de pessoas idosas no Brasil(1).

$\mathrm{Na}$ idade adulta, predominam as doenças crônicas não transmissíveis cuja incidência e mortalidade se elevam à medida que aumenta a vida média da população. Uma das doenças mais temidas é o câncer e, segundo os dados do Instituto Nacional do Câncer (INCA), cerca de 75\% das neoplasias são encontradas em indivíduos com mais de 60 anos. O aumento da expectativa de vida não só eleva a exposição do indivíduo aos fatores de risco presentes no meio ambiente e o tempo dessa exposição, como também o envelhecimento propicia o surgimento de neoplasias genéticas cujo aparecimento é tardio ${ }^{(2)}$.

As alterações da integridade física e emocional por desconforto, dor, fadiga, desfiguração, dependência e perda da autoestima são relatadas pelas pessoas com câncer, que percebem a qualidade de suas vidas profundamente alterada, num curto período de tempo ${ }^{(3)}$.

A fadiga é um sintoma prevalente na doença oncológica, manifestando-se entre $75 \%$ e $95 \%$ dos doentes. Ela compromete as atividades da vida diária, podendo limitá-las e ocasionar prejuízos à qualidade de vida. Os sintomas depressivos, por exemplo, também frequentes nos pacientes oncológicos submetidos à quimioterapia, correlacionam-se com a qualidade de vida dos indivíduos afetados, sobretudo com o domínio "humor" e com o domínio "fadiga"(4).

Fadiga pode ser definida como "uma sensação física desagradável, com sintomas cognitivos e emocionais, descritos como cansaço, que não é aliviado com o emprego de estratégias usuais de restauração da energia. A fadiga varia em duração e intensidade, e reduz em diferentes graus a habilidade para desenvolver as atividades da vida diária", conforme é revelado em estudo no qual foram analisados os instrumentos comumente utilizados na literatura internacional ${ }^{(5)}$.

Nos clientes oncológicos, a fadiga tem sido atribuída a diversas causas, tais como: estado hipermetabólico associado com o crescimento tumoral, competição entre o organismo e o tumor por nutrientes, efeitos deletérios da quimioterapia e da radioterapia, ingesta nutricional inadequada associada à náusea e vômitos decorrentes da terapêutica antineoplásica, anemia, distúrbio do sono e a incerteza e medo quanto ao futuro e a morte ${ }^{(6)}$. Os efeitos terapêuticos e tóxicos dos quimioterápicos dependem do tempo de exposição, da concentração plasmática e da droga utilizada. Seus efeitos colaterais mais frequentes são: mielodepressão, alopecia e alterações gastrintestinais ${ }^{(7)}$.

Avaliar a fadiga "é crucial para identificação dos fatores preditivos, e identificar pacientes fatigados é um desafio, pois fadiga é um sintoma complexo, multidimensional e subjetivo". Depressão, capacidade funcional e prejuízo do sono foram fatores preditivos da fadiga em um estudo realizado com 157 pacientes com câncer ${ }^{(6)}$. Em outro estudo, cujas participantes foram 182 mulheres com câncer de mama, identificou-se a presença de fadiga em 94 mulheres de acordo com a Escala de Fadiga de Piper ${ }^{(8)}$.
Ressalta-se a importância da identificação da fadiga na prática clínica, a qual constitui um diagnóstico de enfermagem encontrado na taxonomia da Nanda International (NANDA-I) e, como tal, deve gerar intervenções de enfermagem que possam auxiliar o paciente oncológico a enfrentar este sofrimento. Um estudo nacional ${ }^{(9)}$ mostrou que o enfermeiro está apto a reconhecer sinais e sintomas de fadiga, os quais constituem as características definidoras propostas pela NANDA-I. Mas, embora identifique tais características e as registre na avaliação do paciente oncológico hospitalizado, o enfermeiro não consegue traduzi-las na elaboração do correspondente diagnóstico de enfermagem e nem gerar as desejadas propostas de intervenção ${ }^{(9)}$. Esse fato pode estar ligado, entre outros fatores, à escassez de estudos que discutam a prevalência desse sintoma complexo em diferentes tipos de pacientes, com destaque para os oncológicos, e que assim despertem reflexões sobre a responsabilidade do enfermeiro no seu manejo. Portanto, estudos devem ser realizados no sentido de alertar os enfermeiros para a identificação e o manejo deste desconforto tão presente no paciente com câncer.

Autores apontam que as estratégias de intervenção para a fadiga podem ser educativas e que há premente necessidade de pesquisas para se desenvolver uma prática clínica baseada em evidências ${ }^{(10)}$.

Deve-se considerar também que os estudos sobre fadiga são realizados, na maior parte, com pessoas adultas, e muito menos frequentemente com idosos. Estes, sabidamente, possuem singularidades inerentes à sua idade que devem ser observadas e respeitadas pelos profissionais que os assistem.

Desta forma, este estudo teve como objetivo identificar a presença e intensidade da fadiga em idosos com diagnóstico de câncer, em tratamento quimioterápico ambulatorial.

\section{MÉTODO}

Pesquisa com delineamento transversal da qual participaram 140 idosos com câncer que realizavam quimioterapia e que atendiam aos seguintes critérios de inclusão: 60 anos ou mais, diagnóstico de câncer confirmado, tratamento com quimioterapia em andamento e participação voluntária no estudo. Os critérios de exclusão observados foram: câncer no sistema nervoso central ou metástases no mesmo, índice de Karnofsky ${ }^{(11)}$ inferior a 60, pós-operatório recente (até 30 dias), condições clínicas (tais como mucosite, dor intensa, dispnéia, náuseas, vômitos) e emocionais (tais como choro, apatia excessiva, agressividade) inadequadas para responder a uma entrevista.

O tamanho amostral foi calculado com apoio de profissional estatístico, considerando-se o valor de 0,50 para o Coeficiente de Correlação de Postos de Spearman, precisão da estimativa de 0,3 e nível de significância alfa de $5 \%{ }^{(12)}$. Acrescentou-se a esta estimativa inicial cerca de $20 \%$ a mais de casos, com a finalidade de prevenir eventuais perdas e obter maior poder para outras análises não planejadas previamente. O número final desejado para a amostra foi de 140 pacientes.

A coleta de dados foi realizada em dois Ambulatórios de Quimioterapia de um hospital universitário do município de Campinas, interior do estado de São Paulo, em dias e horários 
previamente agendados com os profissionais responsáveis, de agosto a dezembro de 2010.

Os dados foram coletados durante a infusão da quimioterapia, após aceitação da participação no estudo e assinatura do Termo de Consentimento Livre e Esclarecido (TCLE). O estudo foi aprovado pelo Comitê de Ética em Pesquisa da Universidade Estadual de Campinas, e foi homologado em agosto de 2009, pelo Parecer 693/2009, CAAE n. 05540146000-09.

Dois instrumentos, apresentados a seguir, foram empregues na coleta de dados, sob a forma de entrevista com cada participante. O primeiro deles, o Questionário de Caracterização Sociodemográfica e Clínica, foi elaborado especificamente para este estudo, com a finalidade de registrar os dados sócio-demográficos e clínicos dos participantes, e foi avaliado por juízes para validação de conteúdo. O segundo, a Escala de Fadiga de Piper-revisada é um instrumento multidimensional para avaliação da fadiga, validado para uso no Brasil ${ }^{(11)}$. Esta Escala contém 22 itens distribuídos em três dimensões: Comportamental (itens 02 a 07) relacionada à capacidade funcional, incluindo questões pessoais, atividades sociais e relacionamento sexual; Afetiva (itens 08 a 12), que busca encontrar o significado atribuído à fadiga; Sensorial/psicológica (itens 13 ao 23), que busca componentes da auto-percepção, emocionais e cognitivos na presença da fadiga. Cada dimensão, assim como o escore total, recebe uma pontuação que corresponde à média dos escores de cada item, e varia de zero a dez. O ponto de corte adotado para considerar a presença de fadiga foi o escore quatro (escore igual ou inferior a quatro, sem fadiga; superior a quatro, com fadiga).

Na Escala de Piper-revisada, além dos 22 itens pontuados, há cinco questões abertas (itens 1, e 24 a 27) que não são usadas para cálculo do escore do instrumento, mas permitem obter dados adicionais como a duração da fadiga, o que o indivíduo acredita causar a fadiga e a presença de outros sintomas, enriquecendo a qualidade das informações. A composição desta escala com múltiplas dimensões é uma característica importante, pois permite o reconhecimento da expressão da fadiga na vida do indivíduo ${ }^{(11)}$.

Conforme orientação obtida diretamente com a pesquisadora responsável pela validação do instrumento, antes da aplicação da escala indagava-se se o idoso sentia fadiga e lia-se para o mesmo uma definição padronizada do sintoma. Os idosos que negaram sentir fadiga não responderam à Escala de Piper-revisada.

Os dados obtidos foram codificados e digitados em planiIha do programa para computador Excel for Windows (Microsoft Inc.), e posteriormente transportados aos softwares SPSS (versão 17.0) e SAS (versão 9.1.3, SAS Institute Inc., Cary, NC, USA, 2002-2003), que foram utilizados na análise dos dados.

Os dados foram analisados com uso da estatística descritiva, sendo calculadas frequências absolutas e proporções para as variáveis categóricas e medidas de tendência central, posição e dispersão para as variáveis contínuas. Os dados apresentavam distribuição diferente da normal ao teste de Kolmogorov-Smirnov, portanto um teste não paramétrico, o coeficiente de correlação de postos de Spearman, foi empregue para avaliar a correlação entre as dimensões e os escores finais do instrumento de fadiga, estabelecendo-se o nível crítico de $5 \%$.
A consistência interna da Escala de Piper foi avaliada pelo coeficiente alfa de Cronbach. Nesse coeficiente, obter valores acima de 0,70 indica adequada consistência interna do instrumento.

\section{RESULTADOS}

A caracterização dos 140 idosos participantes do estudo está apresentada na Tabela 1, a seguir.

Tabela 1 - Distribuição dos idosos segundo características sociodemográficas $(n=140)$. Campinas-SP, 2010

\begin{tabular}{|c|c|c|c|}
\hline \multicolumn{2}{|c|}{ Variáveis } & \multirow{2}{*}{$\begin{array}{c}\mathbf{n} \\
67\end{array}$} & \multirow{2}{*}{$\begin{array}{r}\% \\
47,9\end{array}$} \\
\hline Sexo & Masculino & & \\
\hline & Feminino & 73 & 52,1 \\
\hline \multirow[t]{2}{*}{ Cor da pele } & Branco & 115 & 82,1 \\
\hline & Não branco & 25 & 17,9 \\
\hline \multirow[t]{4}{*}{ Estado conjugal } & Casado & 93 & 66,4 \\
\hline & Viúvo & 32 & 22,9 \\
\hline & Separado & 8 & 5,7 \\
\hline & Solteiro & 7 & 5,0 \\
\hline \multirow[t]{4}{*}{ Anos de estudo completos } & Zero a menos de um ano & 27 & 19,3 \\
\hline & 1 a 3 anos & 33 & 23,6 \\
\hline & 4 a 8 anos & 60 & 42,9 \\
\hline & 9 ou mais & 20 & 14,3 \\
\hline \multirow[t]{3}{*}{ Situação de trabalho atual } & Aposentado e inativo & 118 & 84,3 \\
\hline & Aposentado e ativo & 10 & 7,1 \\
\hline & Do lar & 11 & 7,9 \\
\hline \multirow[t]{5}{*}{ Com quem mora atualmente } & Cônjuge & 56 & 40,0 \\
\hline & Cônjuge e filhos & 28 & 20,0 \\
\hline & Filhos & 26 & 18,6 \\
\hline & Outros familiares & 3 & 2,1 \\
\hline & Instituição & 2 & 1,4 \\
\hline Crença/ religião & Sim & 134 & 95,7 \\
\hline \multirow{5}{*}{$\begin{array}{l}\text { Transporte utilizado para } \\
\text { chegar ao Ambulatório de } \\
\text { Quimioterapia }\end{array}$} & Carro do familiar & 66 & 47,1 \\
\hline & Coletivo do governo & 48 & 34,3 \\
\hline & Coletivo público & 17 & 12,1 \\
\hline & Carro próprio & 8 & 5,7 \\
\hline & Outro & 1 & 0,7 \\
\hline \multirow{3}{*}{$\begin{array}{l}\text { Renda mensal individual } \\
(\mathrm{SM})^{*}\end{array}$} & Até 5 SM & 124 & 88,6 \\
\hline & 6-10 SM & 2 & 1,4 \\
\hline & Acima de $10 \mathrm{SM}$ & 14 & 10,0 \\
\hline \multirow[t]{3}{*}{ Renda mensal familiar (SM) } & Até 5 SM & 107 & 76,4 \\
\hline & 6-10 SM & 29 & 20,7 \\
\hline & Acima de 10 SM & 4 & 2,9 \\
\hline
\end{tabular}

\section{Notas:}

*SM: salário-mínimo vigente de agosto a dezembro de 2010 (R\$ 510,00). Lei 12.255, de 15 de junho de 2010. Disponível em http://www.planalto.gov.br/ ccivil/_Ato2007-2010/2010/Lei/ L12255.htm

Os dados clínicos e de estilo de vida e saúde apresentam-se na Tabela 2, a seguir. 
Tabela 2 - Distribuição dos idosos segundo aspectos de saúde estilo de vida. $(n=140)$. Campinas-SP, 2010

\begin{tabular}{|c|c|c|}
\hline Variável & $\mathbf{n}$ & $\%$ \\
\hline Sofre de doença crônica & 65 & 46,4 \\
\hline \multicolumn{3}{|l|}{ Tipo de doença crônica } \\
\hline Hipertensão arterial & 58 & 41,4 \\
\hline Diabetes & 25 & 17,9 \\
\hline Osteoporose & 21 & 15,0 \\
\hline Outra & 12 & 8,6 \\
\hline Consumo de álcool & 15 & 10,7 \\
\hline Consumo de café & 122 & 87,1 \\
\hline Hábito de fumar & 16 & 11,4 \\
\hline Prática de atividade física & 45 & 32,1 \\
\hline \multicolumn{3}{|l|}{ Quantidade de medicamentos em uso } \\
\hline Zero & 31 & 22,1 \\
\hline Um & 60 & 42,9 \\
\hline Dois & 40 & 28,6 \\
\hline Três & 9 & 6,4 \\
\hline Sintoma relacionado à menopausa $(n=73)$ & 5 & 6,8 \\
\hline Dores nos últimos 30 dias & 72 & 51,4 \\
\hline Sede intensa & 93 & 66,4 \\
\hline Boca seca & 93 & 66,4 \\
\hline Náusea & 76 & 54,3 \\
\hline Vômitos & 61 & 43,6 \\
\hline Diarreia & 60 & 42,9 \\
\hline Ferida na boca e gengivas & 42 & 30,0 \\
\hline
\end{tabular}

Tabela 3 - Coeficiente alfa de Cronbach para a Escala de Fadiga de Piper-revisada e suas dimensões $(n=64)$. Campinas-SP, 2010

\begin{tabular}{lc}
\hline \multicolumn{1}{c}{ Escala de Piper-revisada } & Coeficiente alfa de Cronbach \\
\hline Dimensão comportamental & 0,87 \\
Dimensão afetiva & 0,76 \\
Dimensão sensorial/ psicológica & 0,72 \\
Escore total & 0,84 \\
\hline
\end{tabular}

Analisando-se o prontuário dos 140 idosos participantes quanto aos dados clínicos de interesse, verificou-se que os diagnósticos mais freqüentes foram os tumores do aparelho digestivo $(52,1 \%)$ e ginecológico ou mamário $(37,1 \%)$, diagnosticados há, em média, 2,6 anos (desvio padrão 3,4 anos), com mediana de 1,4 anos, variando de um mês a 16,5 anos. Quanto aos dados informados pelos idosos, a maioria realizou ou realiza radioterapia $(52,1 \%)$ e foi submetido à cirurgia relacionada ao tumor $(73,6 \%)$.

Dentre os 140 participantes, $64(45,7 \%)$ responderam afirmativamente quando indagados sobre a presença de fadiga e, portanto, responderam à Escala de Fadiga de Piper-revisada. Aos 78 restantes $(54,3 \%)$, a escala não foi aplicada.

A análise de confiabilidade da Escala de Fadiga de Piper-revisada foi realizada com os dados dos 64 participantes que responderam a esse instrumento. Verificou-se consistência interna adequada para a escala como um todo, bem como para as três dimensões que a compõem. Estes resultados encontram-se na Tabela 3.

A análise de correlação entre os itens da escala, e destes com o escore total, segundo o Teste de Correlação de Postos de Spearman é apresentada a seguir na Tabela 4.

Tabela 4 - Correlação das dimensões da Escala de Fadiga de Piper-revisada entre si e com o escore total $\mathrm{n}=64)$. Campinas-SP, 2010

\begin{tabular}{lccc}
\hline \multicolumn{1}{c}{ Dimensões } & $\begin{array}{c}\text { Dimensão } \\
\text { afetiva }\end{array}$ & $\begin{array}{c}\text { Dimensão } \\
\text { sensorial/ } \\
\text { psicológica }\end{array}$ & $\begin{array}{c}\text { Escore total } \\
\text { de fadiga }\end{array}$ \\
\hline $\begin{array}{l}\text { Dimensão } \\
\text { comportamental }\end{array}$ & 0,211 & $0,347^{*}$ & $0,764^{*}$ \\
& & & \\
Dimensão afetiva & $-0,018$ & $0,363^{*}$ \\
& & & \\
$\begin{array}{l}\text { Dimensão sensorial/ } \\
\text { psicológica }\end{array}$ & - & - \\
\hline $\begin{array}{l}\text { Notas: } \\
\text { * Resultados significativos }(p<0,01) \text { ao teste de Correlação de Postos de Spearman. }\end{array}$
\end{tabular}

Quatro participantes dentre os 64 que responderam à Escala de Fadiga de Piper-revisada obtiveram escore total inferior a 4 , sendo classificados como 'sem fadiga'. Verificou-se, portanto, que a fadiga estava presente em 60 idosos $(42,9 \%)$ dentre os 140 estudados.

A média dos escores obtidos pelos 60 idosos com fadiga mostrou-se superior ao valor médio (5,0 pontos) da escala. O escore mais elevado foi encontrado na dimensão Afetiva. A análise descritiva dos escores total e de cada dimensão da Escala de Fadiga de Piper-revisada encontra-se na Tabela 5.

Os 60 idosos que relataram sentir fadiga atribuíram a causa deste sintoma ao câncer $(64,1 \%)$, à quimioterapia $(32,8 \%)$ e ao tratamento como um todo $(3,1 \%)$. Como fator de alívio, os idosos relataram: dormir $(27 \%)$, rezar $(17 \%)$ e morrer $(12 \%)$, sendo que alguns deles relataram mais de um fator. Uma parcela considerável $(45 \%)$ disse "não ter o que fazer", pois não há alívio para a fadiga. Os idosos a descreveram como "cansaço", "corpo ruim" e "presença de dor". 
Tabela 5 - Análise descritiva do escore total e das dimensões da Escala de Fadiga de Piper-revisada ( $n=60)$. Campinas-SP, 2010

\begin{tabular}{lccccc}
\hline \multicolumn{1}{c}{ Escala de Fadiga de Piper-revisada } & Média & Desvio padrão & Mediana & Variação observada & Variação possível \\
\hline Dimensão comportamental & 5,9 & 2,5 & 5,7 & $1,0-10,0$ & $0,0-10,0$ \\
Dimensão afetiva & 8,3 & 1,7 & 8,8 & $3,6-10,0$ & $0,0-10,0$ \\
Dimensão sensorial/ psicológica & 5,9 & 1,4 & 5,6 & $3,6-9,0$ & $0,0-10,0$ \\
Escore total de fadiga & 6,5 & 1,1 & 6,4 & $4,2-9,3$ & $0,0-10,0$ \\
\hline
\end{tabular}

\section{DISCUSSÃO}

A amostra do presente estudo caracterizou-se por um discreto predomínio numérico do sexo feminino $(52,1 \%)$, assim como em estudo pregresso realizado com pessoas com câncer, em que 54,9\% eram mulheres ${ }^{(13)}$. A incidência do câncer em 2010, para todas as faixas etárias, era estimada em $253.030(51,7 \%)$ casos novos nas mulheres e 236.240 $(48,3 \%)$ nos homens ${ }^{(1)}$.

Verificou-se que $78,6 \%$ dos idosos referiu residir com o cônjuge, os filhos ou com ambos. Este arranjo familiar pode constituir um aspecto positivo, conforme observado em um estudo cujos participantes eram enfermeiras com câncer ginecológico ou mamário, em que aquelas que apresentavam maior apoio e interação social mantinham práticas mais adequadas de cuidado à saúde ${ }^{(14)}$. Pode-se sugerir que o fato de $47,1 \%$ dos idosos vir para a quimioterapia em carro de familiar reforça a presença do apoio da rede familiar.

Salienta-se a elevada prevalência de uma crença ou religião entre os idosos deste estudo, referida por 95,7\% deles. A literatura aponta que, se a força poderosa da mente for invocada e esta estiver aliada à fé religiosa, com o intuito de recuperar-se do agravo, o paciente se sentirá mais feliz e com melhor disposição para enfrentar a doença ${ }^{(15)}$.

Os efeitos adversos relacionados à quimioterapia, relatados pelos pacientes como mais freqüentes (sede intensa, boca seca e náusea) são comumente encontrados na literatura especializada. Tal fato pode ser explicado pela toxicidade dos quimioterápicos administrados, relacionada à não especificidade celular, que podem gerar efeitos como dor, cansaço, náuseas e vômito ${ }^{(16)}$.

A Escala de Fadiga de Piper-revisada obteve, neste estudo, indícios de consistência interna satisfatória, tanto no seu escore final como nas três dimensões que a compõem, conforme estimado pelo coeficiente alfa de Cronbach. Os resultados obtidos nesse coeficiente foram um pouco inferiores aos relatados pelos autores que validaram o instrumento para uso no Brasi $^{(11)}$, mas ainda assim sugerem que o instrumento mediu adequadamente o construto que se pretendia avaliar.

Identificou-se a presença de fadiga em 60 idosos (42,9\%), constituindo um percentual elevado, embora inferior ao de outros estudos com pacientes oncológicos. Em estudos pregressos a fadiga foi encontrada em cerca de $60 \%$ a $79 \%$ dos pacientes com câncer, sendo a queixa mais comum entre eles ${ }^{(17-18)}$.

Em um estudo com 379 pacientes com câncer, cuja idade média era de 62 anos, identificou-se que a fadiga foi o efeito colateral mais frequente relatado após a quimioterapia ${ }^{(19)}$.
Estes autores encontraram relato de fadiga em 301 pacientes, pelo menos por alguns dias, mensalmente, durante a quimioterapia ${ }^{(19)}$, enquanto que, em um outro estudo com 199 idosos institucionalizados, verificou-se que a fadiga foi relatada como 'um cansaço' por $98 \%$ destes ${ }^{(20)}$. Estudos realizados com idosos (idade média de 71 anos) com câncer em tratamento quimioterápico ambulatorial revelou que 99\% destes relatavam fadiga na semana em que realizavam a quimioterapia ${ }^{(21)}$.

Os idosos que, neste estudo, relataram sentir fadiga descreveram-na como cansaço, corpo ruim e presença de dor. A causa atribuída foi o câncer $(64,1 \%)$, a quimioterapia $(32,8 \%$ ) e o tratamento como um todo (3,1\%). Quando relacionada ao câncer, a fadiga pode ser diferente daquela que ocorre como conseqüência do exercício ou estresse; é um sintoma que se desenvolve ao longo do tempo e tem implicações cognitivas, físicas e emocionais, incluindo a redução de energia, aumento da necessidade de repouso, redução da concentração mental e desgaste da motivação ou interesse em realizar atividades diárias ${ }^{(21)}$.

Como fator de alívio, os idosos entrevistados relataram "dormir", "rezar" e "morrer", enquanto que 45\% deles disseram "não ter o que fazer", pois a fadiga não poderia ser aliviada. Este resultado é corroborado pela literatura cujos achados identificaram que a fadiga advinda do câncer não melhora nem cessa com o descanso(21).

Em estudo pregresso realizado com pessoas com câncer e seus oncologistas identificou-se que os profissionais acreditavam que a dor seria o sintoma relacionado ao câncer que mais prejudicava a vida diária de seus pacientes. Os pacientes, entretanto, apontaram a fadiga como o principal sintoma que interferia negativamente em sua qualidade de vida. Além disto, os oncologistas acreditavam que a investigação e o tratamento da fadiga eram negligenciados e deveriam ser melhor conduzidos, enquanto que os pacientes relatavam que a fadiga seria um sintoma a ser suportado. Em decorrência, 50\% dos pacientes não discutiam este sintoma com os profissionais e nem buscava opções de tratamento ${ }^{(18)}$.

Desta forma, é importante salientar que tanto as limitações dos profissionais para identificação da fadiga quanto a relutância dos pacientes em expressarem suas queixas resultam em subnotificação, que contribui para o desconhecimento da sua prevalência ${ }^{(21)}$.

Alguns autores estimam que até $50 \%$ dos idosos em geral sofrem de fadiga leve. As causas variam, mas a teoria que predomina traz a fadiga como consequência secundária à doença e à medicação. Devido ao seu potencial de comprometimento negativo da vida diária e da qualidade de vida e bem estar do 
paciente, este sintoma deve ser investigado e realizadas intervenções para minimizar seus efeitos ${ }^{(21)}$.

Neste estudo, a dimensão com escore mais elevado dentre as que compõem a Escala de Fadiga de Piper-revisada foi a Afetiva, que é relacionada ao significado atribuído pelo paciente à fadiga, em sua vida, no momento da entrevista. Outros autores verificaram, nesse sentido, que a depressão mostrou-se um relevante fator preditivo de fadiga em pacientes com câncer colorretal, aumentando em quatro vezes o risco de apresentar o sintoma ${ }^{(11)}$.

Deve-se considerar também que os resultados das dimensões Comportamental e Sensorial/Psicológica foram superiores ao ponto médio da escala, indicando sua relevância clínica $^{(11)}$. Estas dimensões podem sofrer o impacto de outros sintomas físicos associados à fadiga, tais como dor, cansaço, náuseas e vômitos, e anorexia, oriundos da toxicidade das drogas quimioterápicas administradas ${ }^{(16)}$, os quais reduzem a capacidade funcional e prejudicam a qualidade de vida ${ }^{(16)}$, com expressiva repercussão emocional.

É preciso estar alerta também para o fato de que, no Brasil, grande parcela das pessoas com 60 anos ou mais relata a percepção de seu estado de saúde como "muito boa" ou "boa", a despeito da presença de mais de uma doença crônica ${ }^{(22)}$. Ou, muitas vezes, o idoso não tem motivação ou coragem de relatar seus sintomas considerando-os "normais", relacionando-os ao processo de envelhecimento e, portanto, interpretados como inevitáveis. Essa postura deve ser desencorajada pelos profissionais de saúde, destacando-se aqui, os enfermeiros que não devem permiti-la se objetivam o alcance de um cuidado de qualidade e uma Enfermagem de excelência. Estes pacientes devem ser estimulados a relatar fadiga caso a sintam.
Algumas limitações deste estudo devem ser observadas. O delineamento da pesquisa é transversal, com dados coletados uma única vez, impedindo o acompanhamento do sintoma ao longo do tempo. As variáveis clínicas se detiveram principalmente naquelas de natureza física, sendo que a literatura traz, em pacientes com câncer, a relevância de variáveis psicológicas como a presença de depressão, que interfere na presença de fadiga. Tais limitações podem ser superadas em estudos futuros longitudinais. Salienta-se que os estudos com esta casuística ainda são insuficientes no Brasil, destacando-se a necessidade de pesquisas clínicas em enfermagem nesta área.

\section{CONCLUSÕES}

A prevalência de fadiga entre os 140 idosos com câncer, submetidos à quimioterapia ambulatorial, participantes deste estudo, foi elevada, sendo identificada em 42,9\% da amostra. Os escores obtidos em todas as dimensões, bem como o escore total da Escala de Fadiga de Piper-revisada são indicativos da relevância da fadiga para esses idosos, pois mostraram-se superiores ao ponto médio da escala. A dimensão Afetiva foi a que apresentou maior impacto, de acordo com a pontuação obtida, implicando na interpretação negativa do idoso a respeito desse sintoma.

A identificação da fadiga como problema relevante nos pacientes idosos oncológicos deverá direcionar a atenção dos profissionais no sentido do seu adequado reconhecimento, inclusive como um diagnóstico de enfermagem. A interpretação negativa que o idoso atribui ao sintoma, embora nem sempre se manifeste em forma de queixa espontânea, também deverá contribuir para a maior preocupação com as intervenções de enfermagem que visem o adequado manejo desse problema.

\section{REFERÊNCIAS}

1. Ministério da Saúde (BR). Secretaria de Atenção à Saúde, Instituto Nacional de Câncer, Coordenação de Prevenção e Vigilância de Câncer. Estimativa 2010: incidência de câncer no Brasil [Internet]. Rio de Janeiro (RJ): INCA; 2009. [acesso em 08 de julho de 2012]. Disponível em: http:// www2.inca.gov.br/wps/wcm/connect/agencianoticias/ site/home/noticias/2009/lancamento_estimativa_2010

2. Nordhturft $V$, Schneider JM, Hebert P, Bradham DD, Bryant DD, Phillips $M$, et al. Chronic disease self-management: improving health outcomes. Nurs Clin North Am [Internet]. 2000 [cited 2012 July 08];35(2):507-18. Available from: http://www.ncbi.nlm.nih.gov/pubmed/10873263

3. Michelone APC, Santos VLCG. Quality of life of cancer patients with and without an ostomy. Rev Latinoam Enferm [Internet]. 2004 [cited 2012 July 08];12(6):34-47. Available from: http://www.scielo.br/scielo.php?script=sci_artt ext\&pid $=$ S0104-11692004000600005

4. Coelho FMR, Sawada NO. A fadiga nos pacientes com câncer de laringe. Rev Latinoam Enferm. 1999;7(5):103-10.
5. Mota DDCF, Cruz DALM, Pimenta CAM. Fadiga: uma análise de conceito. Acta Paul Enferm [Internet] 2005 [acesso em 08 de julho de 2012];18(3):285-93. Disponível em: http://www.scielo.br/pdf/ape/v18n3/a09v18n3.pdf

6. Mota DDCF, Pimenta CAM. Fadiga em pacientes com câncer avançado: conceito, avaliação e intervenção. Rev Bras Cancerol [Internet]. 2002 [acesso em 08 de julho de 2012];48(4):577-83. Disponível em: http://www.inca.gov. $\mathrm{br} / \mathrm{rbc} / \mathrm{n}$ 48/v04/revisao3.html

7. Santos CB, Aguiar PHP, Silva MFG, Marino Júnior R. Qualidade de vida pré e pós-cirurgia de pacientes com meningiomas. J Bras Neurocir [Internet]. 2004 [acesso em 08 de julho de 2012];15(3):85-90. Disponível em: http://bases.bireme. br/cgi-bin/wxislind.exe/iah/online/?lsisScript = iah/iah.xis\&s $\mathrm{rC}=$ google \&base $=$ LILACS\&lang $=\mathrm{p} \&$ nextAction $=$ Ink\&exp $r$ Search $=456146 \&$ indexSearch $=$ ID

8. Lamino DA, Mota DDCF, Pimenta CAM . Prevalência e comorbidade de dor e fadiga em mulheres com câncer de mama. Rev Esc Enferm USP [Internet]. 2011 [acesso em 
08 de julho de 2012];45(2):508-14. Disponível em: http:// www.scielo.br/pdf/reeusp/v45n2/v45n2a28.pdf

9. Gorini MIPC, Silva PO, chaves PL, Ercole JP, Cardoso BC. Registro do diagnóstico de enfermagem: fadiga em prontuários de pacientes oncológicos. Acta Paul Enferm [Internet]. 2010 [acesso em 08 de julho de 2012];23(3):354-8. Disponível em: http://www.scielo.br/scielo.php?pid=S0103$21002010000300007 \&$ script $=$ sci_arttext

10. Menezes MFB, Camargo TC. A fadiga relacionada ao câncer como temática na enfermagem oncológica. Rev Latinoam Enferm [Internet]. 2006 [acesso em 08 de julho de 2012];14(3):442-7. Disponível em: http://www.scielo.br/scielo.php?pid = S0104-11692006000300020\&script $=$ sci_arttext

11. Mota DDCF. Fadiga no doente com câncer coloretal: fatores de risco e preditivos [tese]. São Paulo (SP): Escola de Enfermagem, Universidade de São Paulo; 2008.

12. Bonett, DG, Wright TA. Sample size requirements for estimating Pearson, Kendall and Spearman correlations. Psychometrika [Internet]. 2000 [cited 2012 July 08];65(1):23-8. Available from: http://link.springer.com/ article/10.1007/BF02294183\#page-1

13. Mystakidou K, Parpa E, Tsilika E, Pathiaki M, Patirak E, Galanos A, et al. Sleep quality in advanced cancer patients. J Psychosom Res [Internet]. 2007 [cited 2012 July 08];62(2):527- 33. Available from: http://www.ncbi.nlm. nih.gov/pubmed/17467407

14. Silva IT, Griep RH, Rotenberg L. Social support and cervical and breast cancer screening practices among nursing workers. Rev Latinoam Enferm [Internet]. 2009 [cited 2012 July 08];17(4):514-21. Available from: http:// www.scielo.br/scielo.php?pid = S0104-1169200900 0400013\&script $=$ sci_arttext

15. Teixeira JJV, Lefèvre F. Significado da intervenção médica e da fé religiosa para o paciente idoso com câncer Ciênc Saúde Coletiva [Internet]. 2008 [acesso em 08 de julho de 2012];13(4):1247-56. Disponível em: http://www. scielo.br/scielo.php?script = sci_arttext\&pid = S1413$-81232008000400021 \& \operatorname{lng}=p t \& n r$

16. Jorge LLR, Silva SR. Avaliação da qualidade de vida de portadoras de câncer ginecológico, submetidas à quimioterapia antineoplásica. Rev Latinoam Enferm [Internet]. 2010 [acesso em 08 de julho de 2012];18(5):[07 telas]. Disponível em: http://www.scielo.br/pdf/rlae/v18n5/pt_03.pdf

17. Moore P, Dimsdale, JE. Opioids, sleep, and cancer-related fatigue. Med Hypotheses [Internet]. 2002 [cited 2012 July 08];(58):77-82. Available from: http://www.ncbi.nlm.nih. gov/pubmed/11863402

18. Vogelzang NJ, Breitbart W, Cella D, Curt GA, Groopman JE, Horning SJ, et al. Patient, caregiver, and oncologist perceptions of cancer-related fatigue: results of a tripart assessment survey. Semin Hematol [Internet]. 2000 [cited 2012 July 08];34(3):4-12. Available from: http://www. ncbi.nlm.nih.gov/pubmed/9253778

19. Curt GA, Breitbart W, Cella D, Groopman JE, Horning SJ, Itri LM, et al. Impact of cancer-related fatigue on the lives of patients: new findings from the Fatigue Coalition. Oncologist [Internet]. 2000 [cited 2012 July 08];(5):353-60. Available from: http://www.ncbi.nlm.nih.gov/pubmed/11040270

20. Liao S, Ferrell BA. Fatigue in an older population. J Am Geriatric Soc [Internet]. 2000 [cited 2012 July 08];(48):426-30. Available from: http://www.ncbi.nlm. nih.gov/pubmed/10798471

21. Tralongo $P$, Respini $D$, Ferraù $F$. Fatigue and aging. Crit Rev Oncol Hematol [Internet]. 2003;(48):(Suppl)57-64. Available from: http://www.unboundmedicine.com/medline/ citation/14563522/Fatigue_and_aging_

22. Instituto Brasileiro de Geografia e Estatística. Síntese de Indicadores Sociais: uma análise das condições de vida da população brasileira. Rio de Janeiro (RJ): IBGE; 2010 [acesso em 22 de fevereiro de 2011]. Disponível em: http://www. ibge.gov.br/home/estatistica/populacao/condicaodevida/ indicadoresminimos/sinteseindicsociais2010/SIS_2010.pdf 